\title{
Microstructure and mechanical properties of intermetallic $\gamma$-TiAl alloy alloyed with dysprosium
}

\author{
T. I. Nazarova ${ }^{\dagger}$, K. S. Nazarov, S. N. Sergeev, V. M. Imayev, R. M. Imayev \\ †nazarova.ti@mail.ru
}

Institute for Metals Superplasticity Problems RAS, 39 Khalturin St., 450001, Ufa, Russia

\begin{abstract}
In the present work, the effect of alloying with dysprosium on microstructure and compression mechanical properties of a $\beta$-solidifying $\gamma$-TiAl alloy has been studied. For this, ingots of the alloys Ti-45Al-6(Nb,Mo)-0,2B- $x$ Dy (at.\%) with various additions of dysprosium $(x=0,0,1$ and 1 at.\%) were melted. The as-cast alloys were homogenized and then cooled in a furnace. These conditions of the alloys were assumed as the initial cast conditions. Microstructure examination showed that Ti-45Al-6(Nb,Mo)-0,2B and Ti-45Al-6(Nb,Mo)-0,2B-0,1Dy alloys had near lamellar microstructures in the initial conditions. Alloying with 1 at.\% of dysprosium caused a change in the microstructure morphology from near lamellar to mixed lamellarglobular one. The appearance of globular $\gamma$ and $\alpha^{2}$ grains in the alloy alloyed with 1 at.\% of dysprosium resulted in an appreciable refinement of the $\gamma / \alpha_{2}$ colonies. It was revealed that the addition of 1 at. $\%$ of dysprosium to the Ti-45Al-6(Nb,Mo)-0,2B alloy led to changing the phase composition: along with the basic $\gamma, \alpha_{2}$ and $\beta$ (B2)-phases an additional DyAl ${ }_{2}$ phase was detected. Also, oxide particles $\mathrm{Dy}_{2} \mathrm{O}_{3}$ were revealed in both alloys containing dysprosium. The alloys under study were subjected to heat treatment, which included a three-stage anneal. As a result, near lamellar structure was obtained in all alloys. It has been established that the alloys containing dysprosium in the heat treated conditions demonstrated higher values of both the strength and ductility as compared to the alloy free of dysprosium. At $T=800^{\circ} \mathrm{C}$, the compression mechanical properties of the alloys were found to be similar.
\end{abstract}

Keywords: $\gamma$-TiAl alloy, rare earth element, microstructure, heat treatment, mechanical properties.

\section{Микроструктура и механические свойства интерметаллидного $\boldsymbol{\gamma}$-TiAl сплава, легированного диспрозием}

\author{
Назарова Т. И. ${ }^{\dagger}$ Назаров К. С., Сергеев С. Н., Имаев В. М., Имаев Р. М. \\ †nazarova.ti@mail.ru
}

Институт проблем сверхпластичности металлов РАН, ул. Ст. Халтурина 39, 450001, Уфа, Россия

В работе изучали влияние легирования диспрозием на микроструктуру и механические свойства на сжатие интерметаллидного $\beta$-затвердевающего $\gamma$-TiAl сплава. Для этого были выплавлены слитки сплавов Ti-45Al-6(Nb,Mo)-0,2B- $x$ Dy (ат.\%) с различным содержанием диспрозия $(x=0,0,1$ и 1 ат.\%). Слитки сплавов подвергали гомогенизационному отжигу с последующим охлаждением в печи. Данное состояние было принято за исходное литое состояние. Выполненные исследования показали, что микроструктура сплавов Ti-45Al-6(Nb,Mo)-0,2B и Ti-45Al-6(Nb,Mo)-0,2B-0,1Dy в исходном состоянии преимущественно пластинчатая. Введение в сплав 1 ат.\% диспрозия приводит к изменению морфологии микроструктуры от преимущественно пластинчатой к пластинчато-глобулярной. Появление глобулярных $\gamma$ и $\alpha_{2}$-зерен в сплаве с 1 ат.\% диспрозия сопровождается заметным уменьшением размера $\gamma / \alpha_{2}$ колоний. Было показано, что введение 1 ат.\% диспрозия в сплав Ti-45Al-6(Nb,Mo)-0,2B приводит к изменению фазового состава материала: кроме основных $\gamma, \alpha_{2}$ и $\beta(\mathrm{B} 2)$-фаз в сплаве была обнаружена фаза DyAl. Кроме того, в микроструктуре сплавов, содержащих диспрозий, также были обнаружены оксидные частицы $\mathrm{Dy}_{2} \mathrm{O}_{3}$. Для всех исследованных сплавов была выполнена термическая обработка, включавшая в себя трехстадийный отжиг. В результате во всех сплавах была получена преимущественно пластинчатая микроструктура. Установлено, что сплавы, легированные диспрозием, в термически обработанном состоянии демонстрируют при комнатной температуре повышенные прочностные и пластические свойства на сжатие по сравнению со сплавом, не содержащим диспрозий. При $T=800^{\circ} \mathrm{C}$ механические свойства на сжатие трех сплавов оказались близки.

Ключевые слова: $\gamma$-TiAl сплав, редкоземельный элемент, микроструктура, термическая обработка, механические свойства. 


\section{1. Введение}

В настоящее время интерметаллидные сплавы на основе $\gamma$-TiAl фазы (далее $\gamma$-TiAl сплавы), являются перспективными материалами для применения в авиационной технике благодаря их привлекательным свойствам: высокой удельной прочности, жесткости, сопротивлению ползучести при температурах $T=600-800^{\circ} \mathrm{C}$, сопротивлению окислению и горению при температурах до $T=900^{\circ} \mathrm{C}[1-3]$. В интервале температур $T=20-800^{\circ} \mathrm{C}$ удельный модуль упругости этих сплавов выше, чем у никелевых на 30 - 50\% [2]. Предполагается, что в газотурбинном двигателе легкие $\gamma$-TiAl сплавы $\left(\rho \approx 4 \mathrm{\Gamma} / \mathrm{cm}^{3}\right)$ частично заменят жаропрочные тяжелые никелевые сплавы $\left(\rho=8-8,5\right.$ г $\left./ \mathrm{cm}^{3}\right)$, что позволит существенно повысить его удельные силовые характеристики при одновременном снижении расхода топлива, эмиссии углекислого газа и шумности. Промышленному применению сплавов на основе $\gamma$-TiAl фазы препятствуют их недостаточные технологические и эксплуатационные свойства в литом состоянии. Прежде всего, это низкая пластичность, сохраняющаяся вплоть до температур хрупко-вязкого перехода $\left(T=750-800^{\circ} \mathrm{C}\right)$. Вместе с тем, проблема $\gamma$-TiAl сплавов заключается не просто в повышении пластичности, а в достижении сбалансированного комплекса механических свойств: высокой прочности, в том числе усталостной, высокого сопротивления ползучести и окислению при повышенных температурах при приемлемой пластичности и вязкости разрушения при температурах ниже температуры хрупко-вязкого перехода.

На сегодняшний день наиболее сбалансированные механические свойства среди $\gamma$-TiAl сплавов демонстрируют, так называемые TNM сплавы, затвердевающие полностью через $\beta$-фазу и легированные ниобием, молибденом и бором [4-9]. Для дальнейшего улучшения механических свойств TNM сплавов представляет интерес их легирование редкоземельными элементами. Известно [10 - 18], что введение редкоземельных элементов ( $\mathrm{La}, \mathrm{Ce}, \mathrm{Er}, \mathrm{Y}, \mathrm{Nd}$, Dy) в титановые и интерметаллидные $\gamma$-TiAl сплавы, может приводить к улучшению механической обрабатываемости в литом состоянии благодаря измельчению структуры, к повышению жаростойкости и жаропрочности, а в $\gamma$-TiAl сплавах в некоторых случаях - к повышению технологической пластичности при повышенных температурах. Стоит отметить, что систематические данные о влиянии добавок редкоземельных элементов на структуру и механические свойства $\gamma$-TiAl сплавов в литературе практически не представлены.

Целью настоящей работы явилось исследование влияния легирования диспрозием на микроструктуру, включая фазовый состав, и механические свойства на сжатие интерметаллидного сплава Ti-45Al-6(Nb,Mo)-0,2B (ат.\%), относящегося к $\gamma$-TiAl сплавам последнего поколения (TNM сплавам).

\section{2. Материал и методика эксперимента}

Исследовали сплавы следующих химических составов Ti-45Al-6(Nb,Mo)-0,2B, Ti-45Al-6(Nb,Mo)-0,2B-0,1Dy и Ti-45Al-6(Nb,Mo)-0,2B-1Dy (здесь и далее все составы сплавов указаны в ат.\%). Слитки сплавов весом около 30 грамм изготавливали методом аргонно-дуговой плавки на лабораторной установке. Для достижения химической однородности слитки сплавов переплавляли не менее 7 раз. Для приведения слитков сплавов в равновесное состояние их подвергали гомогенизационному отжигу при $T<T_{e}\left(T_{e}-\right.$ температура эвтектоидного превращения). Данное состояние было принято за исходное литое состояние.

Химический состав сплавов оценивали на растровом электронном микроскопе Vega $3 \mathrm{SBH}$, оснащенном приставкой для энергодисперсионного микрорентгеноспектрального анализа (EDX) Oxford instruments.

Микроструктурные исследования проводили на растровом электронном микроскопе (РЭМ) Tescan Mira 3 LHM в режиме обратно-рассеянных электронов (BSE), обеспечивающем фазовый контраст.

Рентгеноструктурные измерения были выполнены на дифрактометре ДРОН-4 с использованием $\mathrm{CoK}_{\alpha}$ излучения. Для индицирования и обработки данных использовали программный пакет X’Pert HighScore Plus.

Температуры фазовых переходов в сплавах определяли с учетом ранее полученных данных [8], а также с помощью дифференциально-сканирующей калориметрии с использованием прибора STA 449F1 Jupiter Netzsch. Нагрев осуществляли со скоростью 20 К/мин в интервале температур от $30^{\circ} \mathrm{C}$ до $1450^{\circ} \mathrm{C}$.

Термическая обработка была выполнена в печи фирмы ATS. Средний размер колоний/зерен определяли методом случайных секущих, объемную долю зерен $\gamma$-фазы - методом Глаголева.

Испытания на сжатие проводили при $T=20$ и $800^{\circ} \mathrm{C}$ с начальной скоростью деформации $\varepsilon^{\prime}=1,2 \times 10^{-3} \mathrm{c}^{-1}$ с использованием испытательной машины Schenck Trebel. Размеры образцов составляли $\varnothing 5 \times 7$ мм. Испытывали по 4 образца на точку при комнатной температуре и по 2 образца на точку при повышенной температуре. Пластичность на сжатие при комнатной температуре определяли до появления на образце первых трещин. При $T=800^{\circ} \mathrm{C}$ деформацию осуществляли на одинаковую степень $\varepsilon=70 \%$ и о пластичности судили, оценивая визуально боковую поверхность деформированных образцов.

\section{3. Результаты и обсуждение}

Микроструктура слитка сплава Ti-45Al-6(Nb,Mo)-0,2B в исходном литом состоянии представлена на рис. 1a. Видно, что она состоит из неравноосных колоний чере-

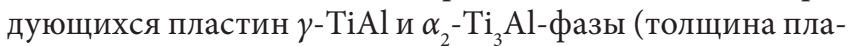
стин не превышает $\lambda=100-150$ нм), а также прослоек светлой $\beta$ (В2)-фазы (В2 - упорядоченная модификация ОЦК $\beta$-фазы). Объемная доля $\beta$ (В2)-фазы не превосходит $2-3 \%$. Вытянутая форма колоний и маленькая толщина $\gamma / \alpha_{2}$ пластин обусловлены относительно высокой скоростью охлаждения слитков в процессе их изготовления [5]. В микроструктуре слитка также наблюдаются бориды (моно- и дибориды титана $[18,19])$, имеющие в основном нитевидную форму. Размер колоний со- 
ставляет в основном $(30-50) \times(20-30)$ мкм. Наличие в сплаве трех фаз - $\gamma, \alpha$ и $\beta(\mathrm{B} 2)$, подтверждается результатами рентгеноструктурного анализа (рис. 2).

Микроструктура сплава, содержащего 0,1 ат.\% диспрозия (рис. 1b) выглядит подобно микроструктуре сплава без диспрозия. Увеличение содержания диспрозия с 0,1 до 1 ат.\% приводит к изменению морфологии микроструктуры и фазового состава сплава (рис. 1с, рис. 3, табл. 1). Видно, микроструктура сплава Ti-45Al-6(Nb,Mo)-0,2B-1Dy трансформировалась из пластинчатой в пластинчатоглобулярную, которая состоит из неравноосных колоний пластин $\gamma$ и $\alpha_{2}$-фазы, окруженных оторочкой $\gamma$-фазы, а также относительно глобулярных микроструктурных составляющих, содержащих смесь $\left(\gamma+\alpha_{2}\right)-$ фаз, разделенные прослойками $\alpha_{2}$-фазы. В микроструктуре сплава также наблюдаются прослойки фазы $\mathrm{DyAl}_{2}, \beta(\mathrm{B} 2)$-фазы и отдельные зерна $\gamma$-фазы. Средний размер колоний пластин составляет $d=31 \pm 2$ мкм. Средний размер глобулярной составляющей $-d=6 \pm 1$ мкм. В обоих сплавах, содержащих диспрозий, были обнаружены частицы $\mathrm{Dy}_{2} \mathrm{O}_{3}$ (рис. 1b, 1c). Диспрозий, обладая высоким сродством к кислороду, по-видимому, способствовал выведению кислорода из матричного сплава с образованием оксида $\mathrm{Dy}_{2} \mathrm{O}_{3}[10,15,17]$.

Для определения температур фазовых превращений для исследуемых сплавов была выполнена дифференциально-сканирующая калориметрия. Анализ полученной кривой для сплава Ti-45Al-6(Nb,Mo)-0,2B показал следующие точки фазовых превращений: $T_{e}=1189^{\circ} \mathrm{C}$ (эвтектоидная температура), $T_{\alpha}=1316^{\circ} \mathrm{C}$ (температура перехода в однофазную $\alpha$-область) (рис. 4). Легирование сплава 0,1 и 1 ат.\% Dу приводит к смещению температуры начала эвтектоидного превращения в сторону бо́льших температур на 5 и $9^{\circ} \mathrm{C}$, соответственно. Температура перехода в однофазную $\alpha$-область смещается в сторону меньших температур на 22 и $26^{\circ} \mathrm{C}$, соответственно.

На основе полученных данных и ранее выполненной работы [8] были разработаны режимы термической обработки сплавов с различным содержанием диспрозия, которые включали в себя трехстадийный отжиг: 1) при $T=T_{\alpha}-15^{\circ} \mathrm{C}(\tau=30$ мин) с последующим охлаждением с печью; 2) при $T=1100^{\circ} \mathrm{C}$ ( $\tau=2$ ч.) с последующим охлаждением с печью и 3 ) при $T=900^{\circ} \mathrm{C}(\tau=4$ ч.) с последующим охлаждением с печью. Термическая обработ-
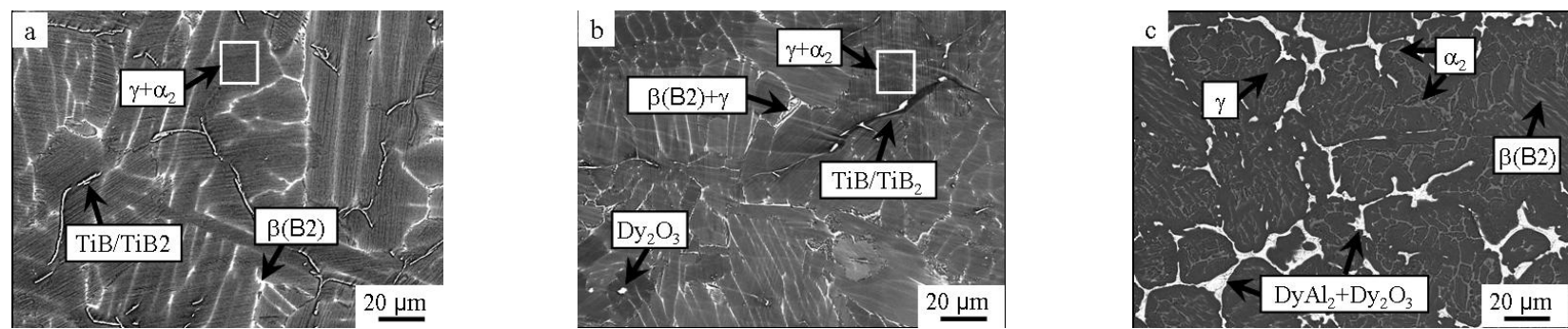

Рис. 1. Микроструктура сплавов (a) Ti-45Al-6(Nb,Mo)-0,2B, (b) Ti-45Al-6(Nb,Mo)-0,2B-0,1Dy, (c) Ti-45Al-6(Nb,Mo)-0,2B-1Dy в исходном литом состоянии (РЭМ, BSE).

Fig. 1. BSE images of the (a) Ti-45Al-6(Nb,Mo)-0,2B, (b) Ti-45Al-6(Nb,Mo)-0,2B-0,1Dy, (c) Ti-45Al-6(Nb,Mo)-0,2B-1Dy alloys in the initial cast conditions.

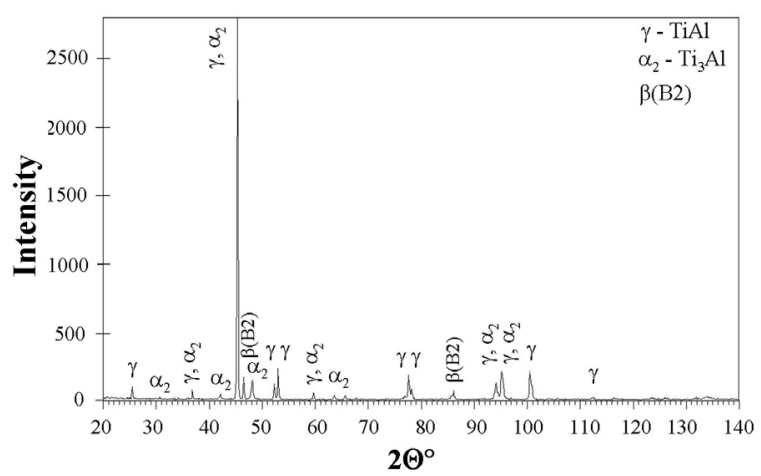

Рис. 2. Дифрактограмма сплава Ti-45Al-6(Nb,Mo)-0,2B в исходном литом состоянии.

Fig. 2. X-Ray diffractogram obtained for the Ti-45Al-6(Nb,Mo)-0,2B alloy in the initial condition.

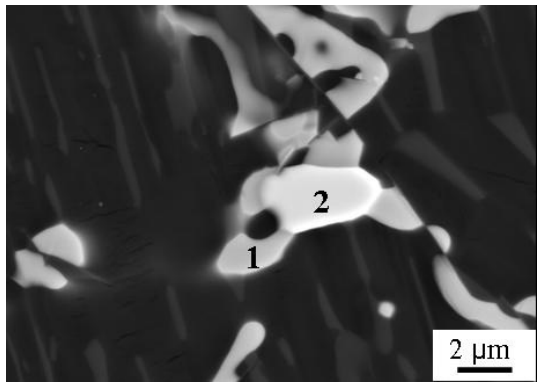

Рис. 3. Микроструктура сплава Ti-45Al-6(Nb,Mo)-0,2B-1Dy в исходном литом состоянии (РЭМ, BSE). Цифрами $(1,2)$ отмечены области, от которых был выполнен EDX анализ.

Fig. 3. BSE image of the Ti-45Al-6(Nb,Mo)-0,2B-1Dy alloy in the initial condition. The areas $(1,2)$ are marked from which the quantitative EDX measurements were made.

Табл. 1. Результаты количественного EDX анализа, полученные от областей, показанных на рис. 3.

Table 1. The results of EDX measurements obtained from the areas marked in Fig. 3 (at.\%).

\begin{tabular}{|c|c|c|c|c|c|}
\hline \multirow{2}{*}{ Участок / Area } & \multicolumn{4}{|c|}{ Элемент, ат.\% / Element, at.\% } & \multirow{2}{*}{ Фаза / Phase } \\
\cline { 2 - 4 } & $\mathrm{Dy}$ & $\mathrm{Al}$ & $\mathrm{O}$ & $\mathrm{Ti}$ & \\
\hline 1 & 20,24 & 54,48 & 5,86 & 17,54 & $\mathrm{DyAl}_{2}[14,20]$ \\
\hline 2 & 35,16 & 1,38 & 60,86 & 2,59 & $\mathrm{Dy}_{2} \mathrm{O}_{3}[10,15,17]$ \\
\hline
\end{tabular}




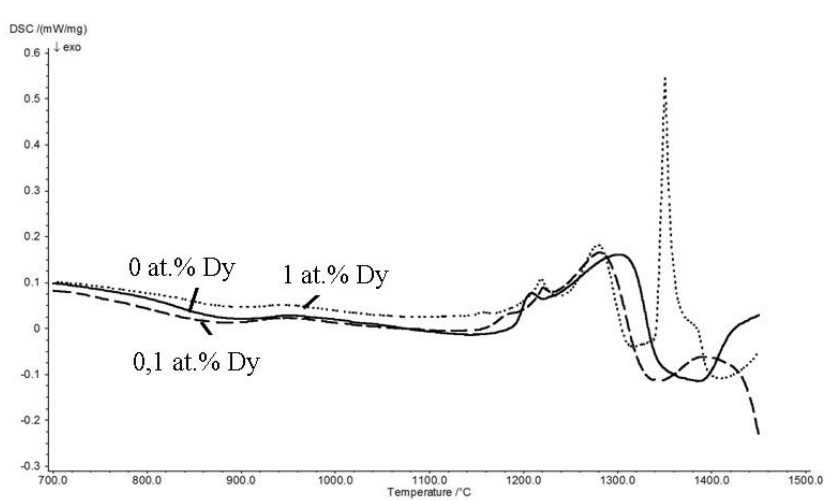

Pис. 4. Кривые дифференциально-сканирующей калориметрии сплавов (a) Ti-45Al-6(Nb,Mo)-0,2B, (b) Ti-45Al-6(Nb,Mo)-0,2B-0,1Dy, (c) Ti-45Al-6(Nb,Mo)-0,2B-1Dy, полученные для исходного литого состояния при нагреве. Скорость нагрева -20 K/мин.

Fig. 4. DTA results obtained for the (a) Ti-45Al-6(Nb,Mo)-0,2B, (b) Ti-45Al-6(Nb,Mo)-0,2B-0,1Dy, (c) Ti-45Al-6(Nb,Mo)-0,2B-1Dy alloys in the initial condition. The heating rate is $20 \mathrm{~K} / \mathrm{min}$.

ка была нацелена, прежде всего, на совершенствование микроструктуры: получение более равновесной микроструктуры пластинчатого или пластинчато-глобулярного типа, сохранение малого размера колоний, увеличение толщины $\gamma / \alpha_{2}$ пластин, а также на растворение $\beta(\mathrm{B} 2)$-фазы.

Видно, что термическая обработка во всех трех сплавах приводит к формированию преимущественно пластинчатой микроструктуры (рис. 5). В случае сплава Ti-45Al-6(Nb,Mo)-0,2B по границам колоний наблюдаются отдельные прослойки/зерна $\gamma$-фазы, $\beta(\mathrm{B} 2)$-фаза практически отсутствует. Объемная доля прослоек/зерен $\gamma$-фазы составляет около 6\%, средний размер колоний $-d=50 \pm 4$ мкм, толщина пластин $-\lambda=70-900$ нм.

При введении в сплав Ti-45Al-6(Nb,Mo)-0,2B 0,1 ат.\% диспрозия термическая обработка приводит к уменьшению среднего размера колоний до $d=41 \pm 3$ мкм, формированию по границам колоний прослоек $\beta(\mathrm{B} 2)$-фазы и оторочки относительно глобулярных зерен $\gamma$-фазы. Объемная доля глобулярной $\gamma$-фазы составляет около $16 \%$, содержание $\beta(\mathrm{B} 2)$-фазы, как и в предыдущем случае, незначительное. Толщина пластин составляет $\lambda=70-500$ нм. В сплаве сохраняются мелкие (диаметром не более 2 мкм) оксидные частицы $\mathrm{Dy}_{2} \mathrm{O}_{3}$.

В сплаве, легированном 1 ат.\% диспрозия, термическая обработка приводит к формированию в структуре крупных пластин $\gamma$ и $\beta(\mathrm{B} 2)$-фазы (толщина пластин составляет $\lambda_{\gamma}=1-10$ мкм, $\lambda_{\beta(\text { в2) }}=0,8-7$ мкм), отдельных относительно глобулярных зерен $\gamma$-фазы, при этом прослойки $\mathrm{DyAl}_{2}$-фазы и оксидные частицы $\mathrm{Dy}_{2} \mathrm{O}_{3}$ coxpaняются, как и в исходном литом состоянии. Средний размер колоний пластин составляет $d=62 \pm 8$ мкм, глобулярной составляющей $-d=10 \pm 3$ мкм.

Механические испытания на сжатие при комнатной и повышенной температурах были выполнены для сплавов в термически обработанном состоянии, содержащих 0 ат.\%, 0,1 ат.\% и 1 ат.\% Dy (рис. 6). Видно, что легирование диспрозием сплава Ti-45Al-6(Nb,Mo)-0,2B приводит к повышению предела текучести $\left(\sigma_{1,25}\right)$, предела прочности $\left(\sigma_{\mathrm{B}}\right)$ и пластичности на сжатие $(\varepsilon)$ при комнатной температуре.
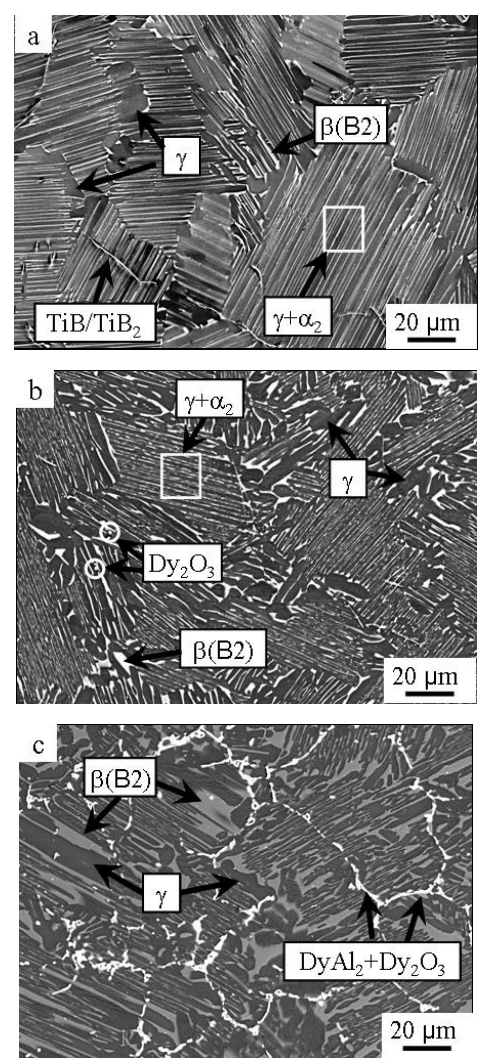

Рис. 5. Микроструктура сплавов (a) Ti-45Al-6(Nb,Mo)-0,2B, (b) Ti-45Al-6(Nb,Mo)-0,2B-0,1Dy, (c) Ti-45Al-6(Nb,Mo)-0,2B-1Dy после термической обработки (РЭМ, BSE).

Fig. 5. BSE images of the (a) Ti-45Al-6(Nb,Mo)-0,2B, (b) Ti-45Al-6(Nb,Mo)-0,2B-0,1Dy, (c) Ti-45Al-6(Nb,Mo)-0,2B-1Dy alloys after heat treatment.
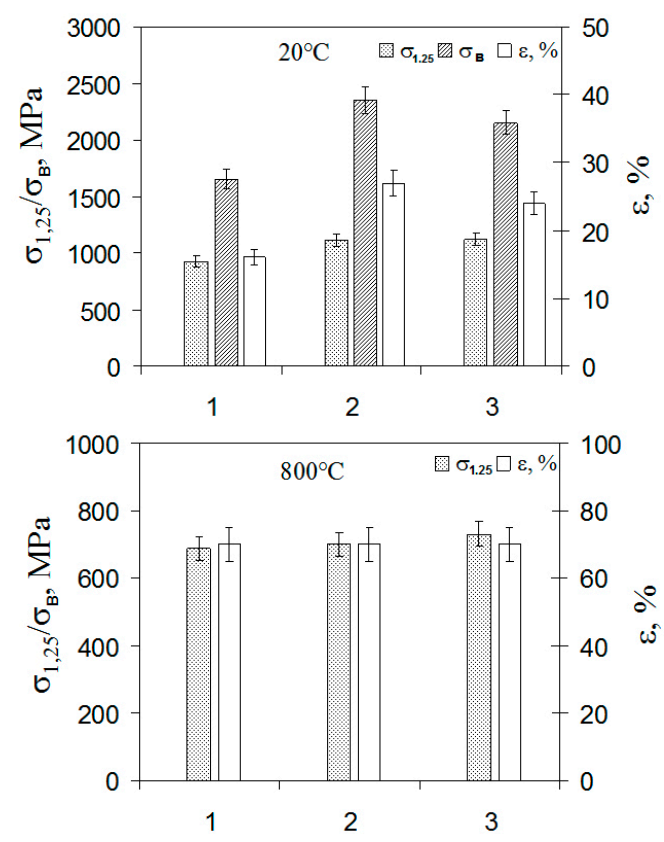

Рис. 6. Механические свойства на сжатие сплавов (1) Ti-45Al-6(Nb,Mo)-0,2B, (2) Ti-45Al-6(Nb,Mo)-0,2B-0,1Dy, (3) $\mathrm{Ti}-45 \mathrm{Al}-6(\mathrm{Nb}, \mathrm{Mo})-0,2 \mathrm{~B}-1 \mathrm{Dy}$ в термически обработанном состоянии $\left(\sigma_{1,25}-\right.$ предел текучести; $\sigma_{\text {в }}-$ предел прочности; $\varepsilon$ - пластичность на сжатие).

Fig. 6. Compression testing results of the (1) Ti-45Al-6(Nb,Mo)-0,2B, (2) Ti-45Al-6(Nb,Mo)-0,2B-0,1Dy, (3) Ti-45Al-6(Nb,Mo)-0,2B-1Dy alloys in heat treated condition $\left(\sigma_{1,25}-\right.$ compression yield strength, $\sigma_{\text {us }}$ - ultimate compression strength; $\varepsilon-$ compression ductility). 
Введение 0,1 ат.\% диспрозия приводит к одновременному повышению прочностных и пластических свойств: предела текучести - с $\sigma_{1,25} \approx 930 \mathrm{MПа} \mathrm{до} \sigma_{1,25} \approx 1110 \mathrm{MПа,}$ предела прочности - с $\sigma_{\text {в }} \approx 1650$ МПа до $\sigma_{\text {в }} \approx 2350 \mathrm{MПа,}$ пластичности на сжатие - с $\varepsilon \approx 16 \%$ до $\varepsilon \approx 27 \%$. По всей видимости, это повышение обусловлено уменьшением размера колоний и изменением морфологии микроструктуры от преимущественно пластинчатой к пластинчатоглобулярной. Повышение содержания диспрозия в сплаве до 1 ат.\% приводит к сохранению предела текучести на уровне предела текучести сплава с содержанием диспрозия 0,1 ат.\%, а значения предела прочности и пластичности на сжатие незначительно снижаются ( $\sigma \approx 2150 \mathrm{MПа,}$ $\varepsilon \approx 24 \%)$, при этом оставаясь заметно выше, чем для сплава без диспрозия. По-видимому, упрочняющую роль в этом случае играют прослойки и частицы фаз $\mathrm{DyAl}_{2}$ и $\mathrm{Dy}_{2} \mathrm{O}_{3}$.

При повышенной температуре сплавы с различным содержанием диспрозия обладают близкими прочностными и пластическими свойствами: на боковой поверхности всех деформированных (на степень $\varepsilon=70 \%$ ) образцов наблюдались трещины, а значения предела текучести были примерно одинаковые.

\section{4. Заключение}

1. Введение в сплав Ti-45Al-6(Nb,Mo)-0,2B диспрозия в количестве 0,1 ат.\% в сочетании с трехстадийной термической обработкой приводит к заметному измельчению микроструктуры и изменению ее морфологии от преимущественно пластинчатой к пластинчато-глобулярной. Увеличение содержания диспрозия до 1 ат.\% в сочетании с термической обработкой приводит к дальнейшему изменению морфологии микроструктуры - формированию толстопластинчатой $(\gamma+\beta(\mathrm{B} 2))$ структуры, окруженной прослойками $\beta(\mathrm{B} 2)$-фазы и глобулярными $\gamma$-зернами.

2. Введение в сплав Ti-45Al-6(Nb,Mo)-0,2B диспрозия в количестве 0,1 ат.\% приводит к появлению в сплаве оксидных частиц $\mathrm{Dy}_{2} \mathrm{O}_{3}$. Увеличение содержания диспрозия до 1 ат.\% приводит к изменению фазового состава сплава: в литом и термически обработанном состоянии помимо основных $\gamma, \alpha_{2}$ и $\beta(\mathrm{B} 2)$-фаз, оксидных частиц $\mathrm{Dy}_{2} \mathrm{O}_{3}$, были обнаружены прослойки $\mathrm{DyAl}_{2}$-фазы.

3. Механические испытания на сжатие сплавов в термически обработанном состоянии показали, что легирование сплава $\mathrm{Ti}-45 \mathrm{Al}-6(\mathrm{Nb}, \mathrm{Mo})-0,2 \mathrm{~B}$ диспрозием приводит к одновременному повышению пластических и прочностных свойств сплава при комнатной температуре. При этом установлено, что сплав легированный 0,1 ат.\% Dу демонстрирует наиболее высокий уровень механических свойств при $T=20^{\circ} \mathrm{C}: \sigma_{1,25} \approx 1110$ МПа, $\sigma_{\text {в }} \approx 2350$ МПа, $\varepsilon \approx 27 \%$. Испытания на сжатие при $T=800^{\circ} \mathrm{C}$ каких-либо различий в деформационном поведении сплавов не выявила.

Благодарность/Acknowledgements. Исследование выполнено при финансовой поддержке РФФИ в рамках научного проекта № 16-33-00786 мол_а, а также в рамках государственной программы фундаментальных исследований № АAАA-A17-117041310215-4./The reported study was funded by RFBR according to the research project № 16-33-00786 мол_a and the Program of fundamental researches of Governement Academy of Science No. AAAAA17-117041310215-4

\section{Литература/References}

1. W. Smarsly, H. Baur, G. Glitz, H. Clemens, T. Khan, M. Thomas. Structural Intermetallics, edit. by Hemker K. J., Dimiduk D.M., Clemens H. et al. TMS, Warrendale, PA. 25 (2001).

2. D.M. Dimiduk. Materials Science and Engineering A. 263, 281 (1999).

3. E. N. Kablov. Aviation materials and technologies 6, (2012), p. 7 - 17 (in Russian) [Е. Н. Каблов Авиационные материалы и технологии. 6, 7 (2012)].

4. E. Schwaighofer, H. Clemens, S. Mayer, J. Lindemann, J. Klose, W. Smarsly, V Güther. Intermetallics. 44, 128 (2014).

5. R. Imayev, V. Imayev, M. Oehring, F. Appel. Intermetallics, 15, 451 (2007).

6. H. Clemens, W. Wallgram, S. Kremmer, V. Güther, A. Otto, A. Bartels. Advanced Engineering Materials. 10, 707 (2008).

7. V.M. Imayev, R.M. Imayev, T.I. Oleneva. Letters on Materials. 1 (1), 25 (2011) (in Russian) [В. М. Имаев, Р. М. Имаев, Т.И. Оленева. Письма о материалах. 1 (1), 25 (2011)].

8. V.M. Imayev, R.M. Imayev, T.I. Oleneva, T. G. Khismatullin. Physics of Metals and Metallography 106, 660 (2008) (in Russian) [В. М. Имаев, Р. М. Имаев, Т.И. Оленева, Т.Г. Хисматуллин. Физика металлов и металловедение. 106, 660 (2008)].

9. A.G. Illarionov, A.A. Popov, S. M. Illarionova Metal Science and Heat Treatment. 12, 17 (2015) (in Russian) [А.Г. Илларионов, А.А. Попов, С.М. Илларионова Металловедение и термическая обработка металлов. 12, 17 (2015)].

10. M. Hadi, A. Shafyei, M. Meratian. Materials Science and Engineering A. 624, 1 (2015).

11. Y. Wu, S. K. Hwang, S. W. Nam, N.J. Kim. Scripta Materialia. 48, 1655 (2003).

12. Y. Wu, K. Hagihara, Y. Umakoshi. Intermetallics. 12, 519 (2004).

13. Y. Chen, F. Kong, J. Han, Z. Chen, J. Tian. Intermetallics. 13, 263 (2005).

14. M. Bulanova, I. Fartushna, K. Meleshevich, A. Samelyuk. Journal of Alloys and Compounds. 598, 61 (2014).

15. S. M. L. Sastry, P. J. Meschter, J.E. O’neal. Metallurgical and Materials Transactions A. 15, 1451 (1984).

16. S. M. L. Sastry, T. C. Peng, L. P. Beckerman. Metallurgical and Materials Transactions A. 15, 1465 (1984).

17. A.I. Khoreev. Vestnik Mashinostroeniya. 11, 54 (2011) (in Russian) [А.И. Хореев. Вестник машиностроения. 11, 54 (2011)].

18. M. Oehring, A. Stark, J.D. H. Paul, T. Lippmann, F. Pyczak. Intermetallics. 32, 12 (2013).

19. U. Hecht, V. Witusiewicz, A. Drevermann, J. Zollinger. Intermetallics. 16, 969 (2008).

20. H. Zhou, W. Liu, S. Yuan, J. Yan. Journal of alloys and compounds. 336, 218 (2002). 